\title{
Burkitt's lymphoma with bilateral cavernous sinus and mediastinal involvement in a child
}

Huisman, Thierry A G M ; Tschirch, Frank ; Schneider, Jacques F L ; Niggli, Felix ; Martin-Fiori, Ernst ; Willi, Ulrich V

\begin{abstract}
We report a 12-year-old boy who presented with incomplete right ophthalmoplegia, exophthalmos and headache. Initial CT and MRI revealed a mass in the right cavernous sinus. During tumour work-up, CT identified additional tumour within the mediastinum. Biopsy of the mediastinal lesion identified Burkitt's lymphoma. We report on this case because radiologists and clinicians should be alerted to identify sites of primary Burkitt's lymphoma outside of the central nervous system if clinical symptoms indicate, or imaging shows, CNS lesions. Primary CNS involvement in Burkitt's lymphoma is rare
\end{abstract}

DOI: https://doi.org/10.1007/s00247-003-1010-x

Posted at the Zurich Open Repository and Archive, University of Zurich ZORA URL: https://doi.org/10.5167/uzh-155853

Journal Article

Published Version

Originally published at:

Huisman, Thierry A G M; Tschirch, Frank; Schneider, Jacques F L; Niggli, Felix; Martin-Fiori, Ernst; Willi, Ulrich V (2003). Burkitt's lymphoma with bilateral cavernous sinus and mediastinal involvement in a child. Pediatric Radiology, 33(10):719-721.

DOI: https://doi.org/10.1007/s00247-003-1010-x 
Thierry A.G.M. Huisman

Frank Tschirch

Jacques F.L. Schneider

Felix Niggli

Ernst Martin-Fiori

Ulrich V. Willi

\section{Burkitt's lymphoma with bilateral cavernous sinus and mediastinal involvement in a child}

Keywords Brain - Mediastinum • Cavernous sinus $\cdot$ Burkitt's lymphoma $\cdot$ MRI $\cdot$ Child
Received: 28 April 2003

Revised: 12 May 2003

Accepted: 17 May 2003

Published online: 18 July 2003

(C) Springer-Verlag 2003

T.A.G.M. Huisman $(\bowtie) \cdot$ F. Tschirch

J.F.L. Schneider · E. Martin-Fiori

U.V. Willi

Department of Radiology and Magnetic

Resonance Imaging, University Children's

Hospital Zurich, Steinwiesstrasse 75,

8032 Zurich, Switzerland

E-mail: thierry.huisman@kispi.unizh.ch

Tel.: + 41-1-2667110

Fax: + 41-1-2667158

F. Niggli

Department of Paediatrics, University

Children's Hospital Zurich, Zurich,

Switzerland

\begin{abstract}
We report a 12-year-old boy who presented with incomplete right ophthalmoplegia, exophthalmos and headache. Initial CT and MRI revealed a mass in the right cavernous sinus. During tumour work-up, CT identified additional tumour within the mediastinum. Biopsy of the mediastinal lesion identified Burkitt's lymphoma. We report on this case because radiologists and clinicians should be alerted to identify sites of primary Burkitt's lymphoma outside of the central nervous system if clinical symptoms indicate, or imaging shows, CNS lesions. Primary CNS involvement in Burkitt's lymphoma is rare.
\end{abstract}

\section{Introduction}

Burkitt's (non-Hodgkin's) lymphoma is a tumour of B lymphoblasts that can arise from any location within the body. The most common manifestation of non-Hodgkin's lymphoma is enlargement of cervical or other lymph nodes [1, 2]. An extranodal origin occurs in $10-34 \%$ of cases, but is unusual at initial presentation. Untreated patients have a poor prognosis and usually die with widespread organ and lymph node metastases. Burkitt's lymphoma is known to be uncommon in North America and Europe, and is seen in children with an average age of 11.5 years [2]. Primary CNS manifestations are rare in children, and neuro-ophthalmological abnormalities are even rarer $[1,2]$. This report describes a 12-year-old boy with Burkitt's lymphoma who initially presented with a cavernous sinus syndrome. Tumour work-up revealed additional lymphoma within the mediastinum.

\section{Case report}

A 12-year-old boy was referred to our hospital with incomplete right ophthalmoplegia consisting of oculomotor nerve palsy and exophthalmos. Symptoms had begun 10 days earlier with headache, nausea and vomiting. Previous medical history was unremarkable. CT and MRI of the brain demonstrated a homogeneously enhancing mass in the right cavernous sinus extending into the orbital apex (Fig. 1). Bone marrow aspiration biopsy disclosed lymphoma. Additional tumour work-up disclosed large mediastinal tumour masses (Fig. 2). Abdominal US was normal. Mediastinoscopic tumour biopsy revealed Burkitt's lymphoma. Serum Epstein-Barr virus (EBV) antibody titres were positive. Cytogenetic analysis of the tumour cells detected an abnormal clone that contained a $\mathrm{t}(8 ; 14)$ translocation typical for B-cell 
non-Hodgkin's lymphoma. A secondary event was observed that resulted in partial gain of $1 \mathrm{q}$ and partial loss of $16 \mathrm{p}$. Gain of $1 \mathrm{q}$ is a common secondary event in B non-Hodgkin lymphoma.
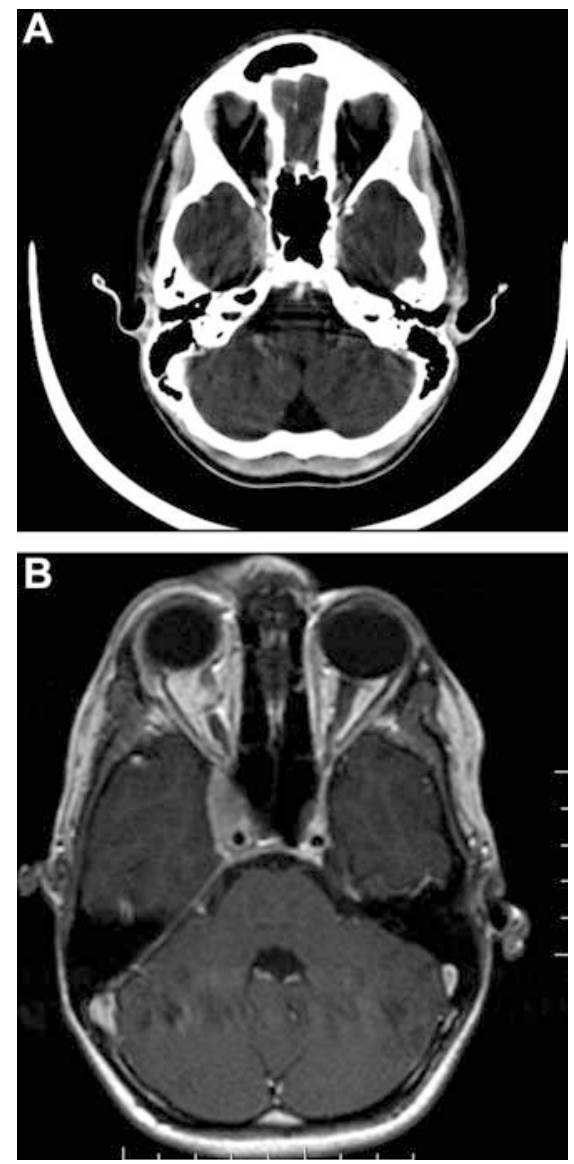

Fig. 1 A Axial contrast-enhanced CT shows enlargement of the right cavernous sinus. B Axial, contrast-enhanced, T1-weighted spin-echo MRI confirms the CT finding. A homogeneously enhancing lesion is seen occupying the entire right cavernous sinus with lateral bowing of the right cavernous sinus border

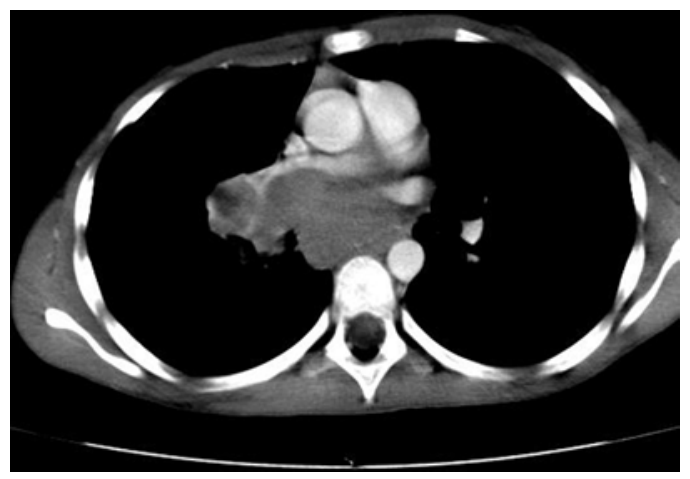

Fig. 2 Contrast-enhanced chest CT at the level of the pulmonary trunk shows a lobulated hypodense mass significantly compressing the right pulmonary artery
Treatment was initiated with high-dose IV and intrathecal chemotherapy according to the NHL-BFM-1995 protocol. Followup imaging showed near complete resolution of the lymphoma masses within the right cavernous sinus and the mediastinum within 2 weeks of chemotherapy. Bone marrow aspiration biopsy was also negative for tumour cells. Clinically, the oculomotor nerve palsy and exophthalmos regressed slightly.

After two additional blocks of chemotherapy, left oculomotor nerve palsy occurred. Imaging showed new tumour within the left cavernous sinus (Fig. 3), as well as mediastinal tumour relapse. Bone marrow aspiration cytology confirmed tumour relapse. In addition, progressive hepatomegaly developed due to tumour infiltration. A final attempt with anti-CD20 antibodies (rituximab) could not prevent further tumour progression. The boy died of progressive liver failure within 3 months of initial diagnosis.

\section{Discussion}

Between 1958 and 1962, Burkitt described an unusual variant of malignant lymphoma in children in subSaharan Africa [2]. Over half of these children presented with jaw tumours. Burkitt's lymphoma is currently known as a tumour developing from B-cells which can arise from any location in the body. Previous studies have suggested that recurrent malaria infection and/or EBV infection in childhood may lead to the development of Burkitt's lymphoma. In the child presented here, serological findings were positive for EBV antibodies.

Primary CNS non-Hodgkin's lymphomas are rare. CNS lymphomas usually result from either direct extension from a primary lesion within the head and neck region or from haematogenous metastases from primary lesions outside the CNS. Cavernous sinus involvement with neuro-ophthalmological symptoms is even rarer. Few cases have been described in the literature $[1,2]$. To our knowledge, this is the third child reported with bilateral cavernous sinus involvement in the literature. More importantly, our case underlines the need to perform complete diagnostic tumour work-up if

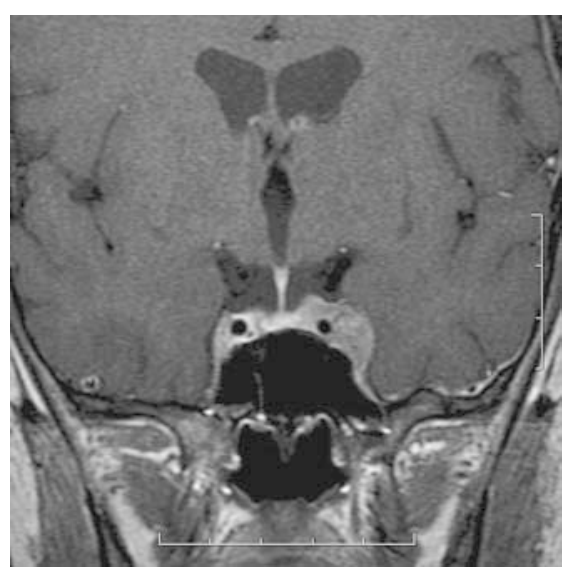

Fig. 3 Follow-up, coronal contrast-enhanced MRI, 2 months after start of chemotherapy shows new tumour within the left cavernous sinus 
bone marrow aspiration in a child with a cavernous sinus mass suggests lymphoma. Other (primary) lymphoma locations should be excluded. The only leading symptom in our child was incomplete ophthalmoplegia. The boy did not suffer from any cardio-pulmonary symptom despite the fact that the right pulmonary artery was significantly compressed by the mediastinal tumour mass.

The rapid course and generalization, with early tumour recurrence and infiltration of bone marrow and liver, despite an intensive pulsed chemotherapy, regimen match previous reports of patients with neurological involvement on initial presentation.

The clinical presentation of incomplete ophthalmoplegia with exophthalmos, known as cavernous sinus syndrome, is due to the involvement of one or multiple cranial nerves (III, IV, first two divisions of V, and VI) that are located within or along the cavernous sinus [3]. Cavernous sinus syndrome can result from multiple neoplastic and non-neoplastic processes that can involve the cavernous sinus. In children, rhabdomyosarcoma, meningioma, juvenile angiofibroma, metastases, pituitary adenoma, infectious and non-infectious cavernous sinus thrombosis, carotid-cavernous fistula, aneurysm of the cavernous carotid artery and Tolosa-Hunt syndrome are among the many lesions that should be excluded by imaging and possibly biopsy $[4,5]$. High-resolution MRI is mandatory so as to exclude pathology within or along the cavernous sinus. In most cases the imaging findings allow characterization of the pathology [5]. If the imaging findings are not conclusive, biopsy may be necessary. If additional tumour is found at less critical anatomical areas, tumour biopsy should be redirected to these regions.

\section{Conclusions}

The cavernous sinus is rarely the primary location for Burkitt's lymphoma. Extensive diagnostic tumour workup should be performed to exclude other (primary) lymphoma locations.

\section{References}

1. Ceyhan M, Erdem G, Kanra G, et al (1994) Lymphoma with bilateral cavernous sinus involvement in early childhood. Pediatr Neurol 10:67-69
2. Kalina P, Black K, Woldenberg R (1996) Burkitt's lymphoma of the skull base presenting as cavernous sinus syndrome in early childhood. Pediatr Radiol 26:416-417

3. Delpassand ES, Kirkpatrick JB (1988) Cavernous sinus syndrome as the presentation of malignant lymphoma. Neurosurgery 23:501-504
4. Eisenberg MB, Al-Mefty O, DeMonte F, et al (1999) Benign nonmeningeal tumors of the cavernous sinus. Neurosurgery 44:949-954

5. Cakirer S (2003) MRI findings in the patients with the presumptive clinical diagnosis of Tolosa Hunt syndrome. Eur Radiol 13:17-28 